\title{
Finite-Difference Time-Domain Modelling of Photonic Crystal Structures
}

\author{
René M. de Ridder and Revco Stoffer \\ University of Twente, MESA Research Institute, Lightwave Devices Group, \\ P.O. Box 217, 7500 AE Enschede, the Netherlands \\ Tel: +3l 53489 2712,Fax:+31534893343,E-mail:R.M.deRidder@el.utwente.nl \\ Kymata Software, P.O. Box 318, 7500 AH Enschede, the Netherlands
}

\begin{abstract}
The usual, highly efficient, modelling tools for planar optical devices are generally not suitable for modelling photonic crystal structures. For example, the Beam Propagation Method fails when applied to these strongly scattering structures since presumptions are made on the propagation direction of the waves. The Finite-Difference Time-Domain method (FDTD), however, as a direct discretisation of Maxwell's equations, does not suffer from such restrictive assumptions. It will be shown that the evolution of the electromagnetic field in both time and space -as calculated using FDTD- can be of considerable help in understanding the physics of photonic crystal structures.
\end{abstract}

Keywords: Photonic Crystals, Photonic Bandgap structures (PBG), Optical waveguide components, Optical filters, Numerical modelling, Finite-Difference Time-Domain methods (FDTD).

\section{INTRODUCTION}

Photonic Crystals are structures having a periodic variation of the refractive index in 1,2 or 3 dimensions with a period in the order of the wavelength of the electromagnetic waves for which they are designed. For a good introduction see e.g. $[1,2]$. These structures can be designed to have a photonic bandgap, i.e. a band of frequencies that cannot propagate through it. Although interesting work is done on 3-dimensional photonic crystals, using layer-by-layer fabrication methods, e.g. [3] or self-organized inverted synthetic opals, e.g. [4], most current interest is in quasi 2-dimensional photonic crystal slabs, consisting of a layer of high-index material which is perforated with a regular array of holes filled with air or another low-index material, see e.g. [5]. An important potential application of photonic crystals is the realisation of ultra-compact integrated optics. Wave propagation in the bandgap of a photonic crystal may be possible along a defect, e.g. a narrow region where the crystal structure has been removed, e.g. [6]. Such regions act as waveguiding channels which can have extremely sharp bends (bending radius of the order of a wavelength), allowing very compact folding of waveguides, e.g. [7, 8]. Also, high-quality optical microresonators can be realised as defects in a photonic crystal, which may lead to very compact optical wavelength filters and add-drop multiplexers for opticall communications $[9,10]$. Another important application field is in highly efficient semiconductor laser and LED light sources. The key to the operation of all these devices is suppression of radiation into unwanted directions, where it is blocked by the forbidden band of the surrounding photonic crystal.

The electromagnetic phenomena in photonic crystal structures are rather complicated compared to the more conventional dielectric waveguide devices. This is because of the multiple scattering phenomena which provide ample mode-conversion mechanisms for coupling all possible guided modes and radiation modes, thus easily causing back-reflection and diffraction of waves from a guided mode in a photonic crystal waveguide. Therefore, efficient modelling tools like the Beam Propagation Method (BPM) [11] where prior assumptions are made on the direction of wave propagation are not applicable. An interesting general extension to arbitrary propagation directions is the Finite-Element Time-Domain BPM [12]. Some important methods for calculating electromagnetic scattering properties are given in [13]. When waveguiding in periodic structures is to be analysed, it is often efficient to subdivide the problem into uniform sections for which the eigenmodes can be calculated and then to match the fields at the interfaces between adjacent sections. The resulting method is known as the $\mathrm{Bi}$ directional Eigenmode Propagation (BEP) [14] or a variant of it, the Method of Lines (MoL) [15]. These methods become inefficient when no extended uniform sections can be distinguished, e.g. in the case of photonic crystal lattice built from circular-shaped holes and curved or tapered waveguides.

A very general method which does not rely on any assumptions of propagating modes is the Finite-Difference Time-Domain method. It involves a direct discretisation of Maxwell's equations, so that given a starting field distribution or driving field (e.g. an incident wave), the evolution of this field in space and time can be calculated. A very useful overview of the method with ample attention to implementation details and boundary conditions can be found in a book by Taflove [16]. A drawback of this method is required number of discretisation points required for obtaining stability and accurate results from the algorithm. The spatial step size should be a fraction (less than about a tenth) of the smallest wavelength and the time step should be less than the corresponding fraction of the smallest wave period in the problem. This rather fine calculation grid puts a practical limit on the size of the problems that can be handled on a given computer. The needed processing time is directly 
proportional to the number of grid points $(x, y, z . t)$. A current $1 \mathrm{GHz}$ personal computer can process 1 or $2.10^{10}$ grid points per hour, which limits the two-dimensional analysis of non-resonant structures to a region of up to about 1000 square wavelengths. For analysing the steady state of high-Q resonant structures or 3-dimensional problems, the region should be considerably reduced (or a supercomputer should be used). In the remainder of this paper some results of 2-dimensional calculations on photonic crystal structures will be shown.

\section{BANDGAP CALCULATION}

An intuitively simple method for calculating a bandgap in a photonic crystal is to determine the transmission through the crystal of waves in all directions over a the range of frequencies of interest. At first sight this method seems rather awkward, since it involves calculations for many angles of incidence and many frequencies over a spatial region of, in principle, infinite extent. However, in many practical applications, a photonic crystal is built from materials having a high refractive index contrast (this is necessary in order to obtain an appreciable bandgap), where waves at frequencies in the bandgap will penetrate for only a few periods. Therefore, it is sufficient to look at the transmission through a relatively thin "block" of photonic crystal material in order to determine its bandgap. Also, using the FDTD method, the results for all angles and frequencies can be obtained in a single calculation. A point source in free space generates spherical waves which cover all possible angles of incidence when impinging on the plane boundary of a photonic crystal (for symmetry reasons, only a limited angular range needs to be taken into account). Furthermore, if this point source is excited with a short pulse, then the resulting waves will cover a broad frequency spectrum. At a large number of positions at the far end of the crystal, the transmitted field is determined and using a Fourier transform, its spectrum is calculated. The collection of frequencies which are not transmitted to any of these positions make up the bandgap(s) of the crystal. Visually the bandgap is immediately apparent when all the transmission spectra are superimposed [17].

The method of obtaining frequency spectra by transforming a pulse response from the time domain calculations does not easily account for material dispersion which is specified in the frequency domain. However, a method for incorporating material dispersion in FDTD calculations is known [16]. On the other hand, calculations have shown that in some cases material dispersion may be neglected with respect to the large "geometric" dispersion of the periodic photonic crystal itself [18].

\section{WAVEGUIDE CALCULATIONS}

An important property of a waveguide is the frequency-dependent effective refractive index of the supported mode(s). One way to obtain this dispersion is to perform a modal analysis. However, using the FDTD, the dispersion can be determined without performing a formal modal analysis, as illustrated in Fig. 1. In this example, a waveguide is formed by removing a row of pillars from a photonic crystal consisting of a 2-D square array of square cross-section high-index pillars in air. The field distribution of the wave or waves propagating in the channel is calculated using FDTD. From the spatial pattern the wavelength and hence the phase velocity is found. From its frequency-dependence also the group velocity can be determined. If more than a single mode is excited in the waveguide they can be separated by spatial Fourier decomposition of the field pattern.

Another practically important issue is efficient coupling of waves into a waveguide in a photonic crystal. The FDTD has been successfully applied for calculating and optimising the coupling efficiency both from a freespace Gaussian beam and from a conventional dielectric slab waveguide to a waveguide of the type shown in Fig. la [17].
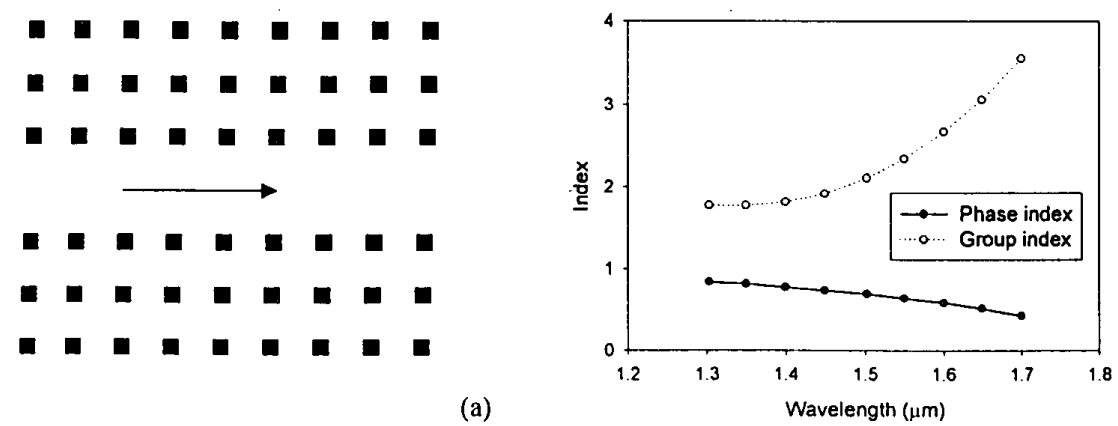

(b)

Figure 1. Calculating waveguide dispersion. (a) The field distribution in the waveguide is calculated. By determining the wavelength of the resulting pattern, the effective (phase) index and from that, the group index can be calculated (b). 


\section{RESONANT CAVITY COUPLED TO PC-WAVEGUIDE}

A simple approach to filtering is coupling a resonant cavity to a straight waveguide in a photonic crystal, as shown in the inset of Fig. 2a. [10]. The configuration is similar to the well-known parallel-connected, shortcircuited waveguide stub in microwave engineering. Inside the band gap, the device has the periodic notch filter response shown in Fig. 2a., as calculated using FDTD. The quality factor of the resonator, and hence the finesse of the filter, can be substantially improved by placing one additional high-index pillar in the entrance of the stub, which reduces the coupling strength between waveguide and cavity (Fig. 2b).

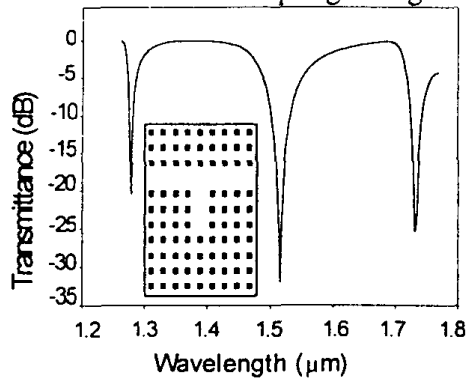

(a)

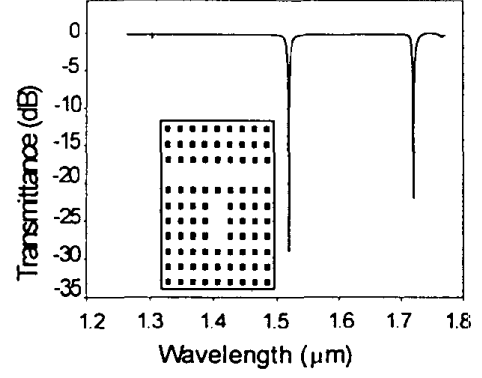

(b)
Figure 2. Optical wavelength filter consisting of cavity resonator, coupled to waveguide in photonic crystal. The graphs show the transfer function (waveguide transmission versus wavelength); the inset in each graph shows the respective configuration.

(a) Strong coupling;

(b) reduced coupling

\section{DIFFRACTION EFFECTS}

The examples shown above have been limited to two-dimensions, i.e. the structure extends to infinity in the direction perpendicular to the plane of calculation. In reality, however, such structures do not exist, e.g., pillars are of finite length and need a support; holes can be etched only down to a certain depth in a layered material structure or in a thin membrane suspended in air. Modelling such quasi-2D structures requires full 3D calculations which may easily exhaust the available computer resources, as mentioned in the introduction.

In the framework of a European cooperation in the COST268 action, an example structure has been defined which allows 2D-calculation of loss due to diffraction out of the photonic-crystal plane. This quasi-1D structure consists of a slab waveguide in which a deep grating having a finite number of periods has been etched (Fig. 3a) [18]. The objective of the study was to compare the transmission, reflection and loss spectra obtained using several modelling methods. For the FDTD calculations, an extremely short pulse of $8 \mathrm{fs}$ duration covering a broad wavelength spectrum centred at $1100 \mathrm{~nm}$, has been launched as a TE mode into the slab waveguide. Figure $3 \mathrm{~b}$ shows the pulse interacting with the grating. Different frequency components are scattered in different

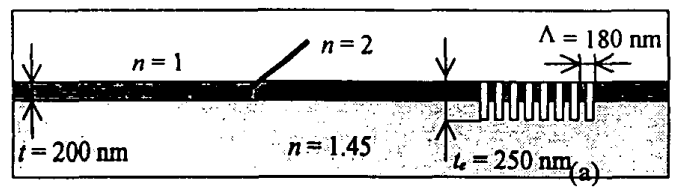

(a)

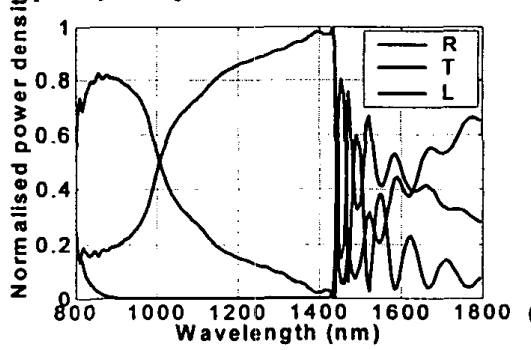

(b)

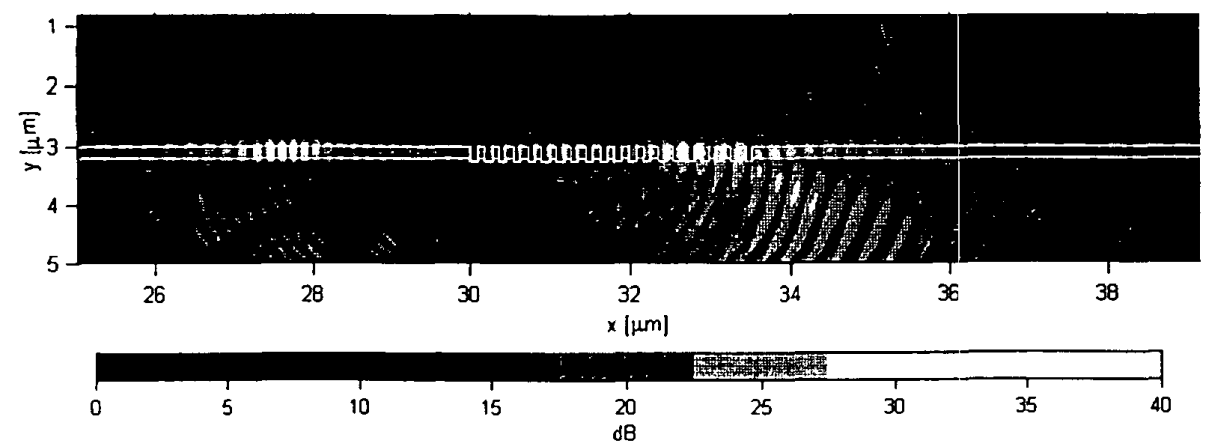

(c)

Figure 3. Diffraction effects from 20-period deep grating in silicon nitride slab waveguide on $\mathrm{SiO}_{2}$. (a) Structure; (b) Transmission, reflection and diffraction of different spectral components of a pulse into different directions; (c) Calculated normalized reflection (R), transmission (T) and diffraction (L) loss spectra. 
directions, or are (almost) fully reflected or transmitted. This process is best observed and interpreted by watching an animation of the field evolution, which has been made available on the internet [19]. Finally, the reflected and transmitted pulses have been Fourier-transformed, in order to obtain their spectra. After normalisation with respect to a pulse that has travelled through a reference slab waveguide without a grating, the reflection and transmission spectra, shown in Fig. 3c, have been obtained. Since we considered lossless materials, the diffraction loss spectrum (also in Fig. 3c) is easily obtained by subtracting the sum of reflection and transmission spectra from unity.

\section{CONCLUSIONS}

It has been shown that the FDTD method is well-suited for modelling many phenomena in photonic crystal structures. Its applications include the calculation of a photonic bandgap, waveguide dispersion, resonator-based filters and diffraction effects. The dynamics of the electromagnetic phenomena can be investigated by studying an animated time-sequence of calculated field distributions. The most important limitations of the FDTD arise from the required computer resources and the difficulty of incorporating material dispersion.

\section{ACKNOWLEDGEMENTS}

The authors would like to thank their colleagues Hugo Hoekstra and Brenny van Groesen for many fruitful discussions. Gerard Klaasse did many of the initial calculations on diffraction effects. The international co-operation on modelling of photonic crystal structures in the framework of the European COST268 and the resulting discussions have proved to be very stimulating for this work. Part of this work was funded by the Dutch Technology Foundation STW.

\section{REFERENCES}

[1] J.D. Joannopoulos, R.D. Meade, J.N. Winn, Photonic crystals: Molding the flow of light, Princeton, NJ: Princeton University Press, 1995.

[2] T.F. Krauss, R.M. De la Rue: Photonic crystals in the optical regime - past, present and future, Prog. Quantum Electron., vol. 23, pp. 5l-96, 1999

[3] S. Noda, K. Tomoda, N. Yamamoto, A. Chutinan: Full Three-Dimensional Photonic Bandgap Crystals at NearInfrared Wavelengths, Science, vol. 289, pp. 604-606, 2000

[4] J.E.G.J. Wijnhoven, W.L. Vos: Preparation of Photonic Crystals Made of Air Spheres in Titania, Science, vol. 281. pp. 802-804. 1998

[5] H. Benisty, C. Weisbuch, D. Labilloy, M. Rattier, C.J.M. Smith, T.F. Krauss, R.M. De la Rue, R. Houdre, U. Oesterle, C. Jouanin, D. Cassagne: Optical and confinement properties of two-dimensional photonic crystals, J. Lightwave Technol., vol. 17, pp. 2063-2077, 1999.

[6] S.G. Johnson, P.R. Villeneuve, S.H. Fan, J.D. Joannopoulos: Linear waveguides in photonic-crystal slabs, Phys. Rev. $B$, vol. 62 , pp. $82 I 2-8222,2000$.

[7] A. Mekis, J.C. Chen, I. Kurland, S. Fan, P.R. Villeneuve, J.D. Joannopoulos: High Transmission through Sharp Bends in Photonic Crystal Waveguides, Phys. Rev. Lett., vol. 77, pp. 3787-3790, 1996.

[8] A. Chutinan, S. Noda: Waveguides and waveguide bends in two-dimensional photonic crystal slabs, Phys. Rev. B, vol. 62, pp. 4488-4492, 2000

[9] S. Fan, P.R. Villeneuve, J.D. Joannopoulos, H.A. Haus: Channel drop tunneling through localized states, Phys. Rev. Lett., vol. 80, pp. 960-963, 1998.

[10] R. Stoffer, H.J.W.M. Hoekstra, R.M. de Ridder, E. van Groesen, F.P.H. van Beckum: Numerical studies of 2D photonic crystals: Waveguides, coupling between waveguides and filters, Optical and Quantum Electronics, vol. 32. pp. 947-96I, 2000.

[11] H.J.W.M. Hoekstra: On beam propagation methods for modeling in integrated optics, Optical and Quantum Electronics, vol. 29, pp. 157-171, 1997.

[12] M. Koshiba, Y. Tsuji, M. Hikari: Time-Domain Beam Propagation Method and its application to photonic crystal circuits, J. Lightwave Technol. vol. 18, pp. 102-110, 2000.

[13] J.B. Pendry: Calculating photonic band structure, J. Phys. [Condensed Matter], vol. 8. pp. 1085-1108, 1996

[14] G.Sztefka, H.-P. Nolting: Bidirectional eigenmode propagation for large refractive index steps, IEEE Photonics Technol. Lett. vol. 5, pp. 554-557, 1993

[15] S.F. Helfert, R. Pregla: Efficient analysis of periodic structures, J. Lightwave Technol. vol. 16, pp. 1694-702, 1998.

[16] A. Taflove: Computational Electrodynamics: The Finite-Difference Time-Domain Method, Boston: Artech House Inc., 1995.

[17] R. Stoffer, R.M. de Ridder, H.J.W.M. Hoekstra, E.W.C. van Groesen, F.P.H. van Beckum, A. Driessen: Calculations on 2-dimensional waveguides in photonic crystals, in Proc LEOS'99 Ann. Meeting. San Francisco, USA, 8$11 / 11 / 1999$, vol. 2. pp. 421-422

[18] J. Čtyroký, S. Helfert, R. Pregla, P. Bienstman, R. Baets, R.M. de Ridder, R. Stoffer, G. Klaasse, J. Petrácek, R. M. De La Rue: Bragg waveguide grating as a ID photonic bandgap structure: COST 268 modelling task, Submitted to Optical and Quantum Electronics, May 2001 .

[19] http://www.el.utwente.nl/tdm/ldg/research/pc_dev/ 